\title{
Influence of bulk dielectric polarization upon PD transients: Effect of multiple dielectric layers
}

\section{McAllister, lain Wilson; Crichton - Fratrådt, George C}

Published in:

Eleventh International Symposium on High Voltage

Engineering, IEE Conference Publication No.467

Publication date:

1999

Document Version

Publisher's PDF, also known as Version of record

Link back to DTU Orbit

Citation (APA):

McAllister, I. W., \& Crichton - Fratrådt, G. C. (1999). Influence of bulk dielectric polarization upon PD transients: Effect of multiple dielectric layers. In Eleventh International Symposium on High Voltage Engineering, IEE Conference Publication No.467 (Vol. 4, pp. 71-74). IEEE.

\section{General rights}

Copyright and moral rights for the publications made accessible in the public portal are retained by the authors and/or other copyright owners and it is a condition of accessing publications that users recognise and abide by the legal requirements associated with these rights.

- Users may download and print one copy of any publication from the public portal for the purpose of private study or research.

- You may not further distribute the material or use it for any profit-making activity or commercial gain

- You may freely distribute the URL identifying the publication in the public portal 
INFLUENCE OF BULK DIELECTRIC POLARIZATION UPON PD TRANSIENTS: EFFECT OF MULTIPLE DIELECTRIC LAYERS

I W McAllister and G C Crichton

Department of Electric Power Engineering, Technical University of Denmark Denmark

\section{ABSTRACT}

A physically valid theory of partial discharge transients has been deve1oped using a field-theoretical approach. The theory is based upon the concept of charge induced upon the detecting electrode by the partial discharge. This induced charge can be resolved into a component associated with the actual space charge in the void and one related to changes in the polarization $\delta \vec{P}$ of the bulk dielectric. These changes are brought about by changes in the field external to the void, which in turn are due to the void space charge. The magnitude of the induced charge component due to $\delta \vec{P}$ is discussed in relation to a three-layer bulk dielectric.

\section{INTRODUCTION}

A partial discharge in a gaseous void leads not only to a charge being induced on the detecting electrode, but also to a change in the polarization of the bulk dielectric. This change $\delta \vec{P}$ is reflected in the charge induced upon the detecting electrode $[1,2]$.

The evaluation of the induced charge can be made using either the $\lambda$ function, or the $\phi$ function [1]. These functions take account of the dielectric polarization either implicitly $(\lambda)$ or explicitly $(\phi)$. Through their use it becomes possible to identify the influence of the change in dielectric polarization upon the induced charge.

Recently the influence of $\delta \vec{P}$ upon the induced charge associated with a two-layer bulk dielectric was examined in detail [2]. The component of the induced charge due to $\delta \vec{P}$ was found to increase or decrease depen- ding upon the ratio of the dielectric permittivities and within which medium the void was located. This increase/decrease is of course reflected in the Poissonian induced charge $q$.

In the present paper, the behaviour of a three-layer dielectric is examined and compared with that of the two-layer system. Although the presence of the third dielectric modifies the level of response, the induced charge characteristics remain similar to those of the two-layer system.

\section{POISSONIAN INDUCED CHARGE}

The charge induced on an electrode can be described in terms of the Poissonian and the Laplacian components [3]. The Poissonian induced charge is that component of the induced charge which is rigidly linked to the space charge source. The Laplacian component is as sociated with any change in electrode potential. These two components are equal in magnitude but opposite in polarity.

The final value of the Poissonian ininduced charge $q$ due to a partial discharge can be mathematically resolved into two components:

$q=q_{\mu}+q_{P}$

where $q_{\mu}$ is the induced charge directly associated with the space charge in the void, while $q_{P}$ represents the induced charge related to the change in the dielectric polarization due to the field of this space charge [1].

With respect to the detecting electrode, the effect of the void wall charges can be considered as the effect of a dipole of moment $\vec{\mu}$ located within the void [4]. The Pois- 
sonian induced charge arising from a dipole is given by

$q=-\vec{\mu} \cdot \vec{\nabla} \lambda$

where $\lambda$ represents the proportionality factor between the charge in the void and the induced charge on the detecting electrode. The $\lambda$ function is a solution of the general Laplace equation [1].

Any method of solving Laplace's equation can be used to determine $\lambda$. The boundary conditions are $\lambda=1$ at the detecting electrode, while at all other electrodes $\lambda=0$. In addition, the condition

$\varepsilon_{+}(\partial \lambda / \partial n)_{+}=\varepsilon_{-}(\partial \lambda / \partial n)$.

must be fulfilled at all dielectric interfaces such as the walls of voids. In (3), $\lambda$ is differentiated in the direction normal to the interface, and the signs + and - refer to each side of the interface, respectively.

However, if the dimensions of the void are such that $\vec{\nabla} \lambda$ may be assumed constant within the void, then we can introduce another function, $\lambda_{0}$, which represents the $\lambda$ function in the absence of the void. As $\lambda$ is a solution of Laplace's equation, then by mathematical analogy with electrostatic fields, the relation between the $\lambda$ and $\lambda_{0}$ functions is given by

$\vec{\nabla} \lambda=h \vec{\nabla} \lambda_{0}$

For the voids under consideration, the parameter $h$ is a scalar which depends on the void geometry and the relative permittivity of the bulk medium in which the void is located. Employing $\lambda_{0}$, the Poissonian induced charge may be expressed as

$q=-h \vec{\mu} \cdot \vec{\nabla} \lambda_{0}$

The component of the Poissonian induced charge related to the void space alone is given by

$q_{\mu}=-\vec{\mu} \cdot \vec{\nabla} \phi$

The proportionality factor $\phi$ is a solution of the reduced Laplace equation [1]. The boundary conditions are $\phi=1$ at the detecting electrode and $\phi=0$ at all other electrodes. Hence from ( 1$),(5)$ and ( 6$)$, the polarization component $q_{P}$ of the Poissonian induced charge may be expressed as

$q_{P}=-\vec{\mu} \cdot\left(h \vec{\nabla} \lambda_{0}-\vec{\nabla} \phi\right)$

THE $\lambda_{0}$ FUNCTION

For a homogeneous dielectric system, $\lambda_{0}$ is a solution of the reduced Laplace equation, and hence in such situations $\lambda_{0}$ and $\phi$ are synonomous. Consequently (7) would reduce to

$q_{P}=-(h-1) \vec{\mu} \cdot \vec{\nabla} \lambda_{0}$

For a heterogeneous dielectric system, $\lambda_{0}$ will usually be a solution of the general Laplace equation. To study this situation, we consider a planar electrode geometry with a three-layer dielectric. In rectangular coordinates, the electrodes are represented by $z=0$ and $z=d$. The dielectric interfaces are then taken as $z=s$ and $z=t$, with $s<t<d$. The permittivity of the layer of thickness $s$ is $\varepsilon_{1}$, that of thickness $(t-s)$ is $\varepsilon_{2}$ while the remaining layer of thickness $(d-t)$ has a permittivity of $\varepsilon_{3}$.

If the lower electrode is used as the detecting electrode, then the boundary conditions for the $\lambda_{0}$ function are $\lambda_{0}=1$ for $z=0$ and $\lambda_{0}=0$ for $z=d$. Thus the $\lambda_{0}$ functions of the three media are given by

$\lambda_{01}=\frac{B}{A}$

for $0 \leq z \leq s$, where

$A=\varepsilon_{1} \varepsilon_{2}(d-t)+\varepsilon_{1} \varepsilon_{3}(t-s)+\varepsilon_{2} \varepsilon_{3} s$

$B=\varepsilon_{1} \varepsilon_{2}(d-t)+\varepsilon_{1} \varepsilon_{3}(t-s)+\varepsilon_{2} \varepsilon_{3}(s-z)$

$\lambda_{02}=\frac{\varepsilon_{1} \varepsilon_{2}(d-t)+\varepsilon_{1} \varepsilon_{3}(t-z)}{A}$

for $s \leq z \leq t$, and 
$\lambda_{03}=\frac{\varepsilon_{1} \varepsilon_{2}(d-z)}{A}$

for $t \leq z \leq d$. The $\lambda_{0}$ subscripts 1,2 and 3 , refer to the particular dielectric layers.

On differentiating with respect to $z$, the relevant expressions for the $\lambda_{0}$ gradients are obtained; viz.

$\vec{\nabla} \lambda_{01}=\frac{-\varepsilon_{2} \varepsilon_{3} \vec{e}}{A}$

$\vec{\nabla} \lambda_{02}=\frac{-\varepsilon_{1} \varepsilon_{3} \vec{e}}{A}$

and

$\vec{\nabla} \lambda_{0,3}=\frac{-\varepsilon_{1} \varepsilon_{2} \vec{e}}{A}$

where $\vec{e}$ is a unit vector in the positive $z$ direction.

For a homogeneous medium $\lambda_{0}=\phi$ and thus for a planar system we have

$\vec{\nabla} \lambda_{0}=\vec{\nabla} \phi=-\frac{\vec{e}}{d}$

This expression can be obtained from (14), (15) or (16) for $\varepsilon_{1}=\varepsilon_{2}=\varepsilon_{3}$.

\section{HETEROGENEOUS DIELECTRIC SYSTEM}

To undertake an assessment of the behaviour of a three-layer dielectric system upon PD transients we will assume that the dipole $\vec{\mu}$, associated with the charge which accumulates at the void wall following a partial discharge, is considered a constant in this study. In addition, it will be assumed that the void is more than 10 times its greatest linear dimension from a dielectric interface, such that the $\vec{\nabla} \lambda$ distribution within the void is effectively uniform: $i$.e. the existence of the interface does not perturb $\vec{\nabla} \lambda$ within the void. This implies that the concept of $h$ is valid and that (4) may be employed.

Variation of $q_{P n}$

With respect to the component of the induced charge related to $\delta \vec{P}$, we have upon combining (7) and (5)
$\frac{q_{P n}}{q_{n}}=\frac{-\vec{\mu} \cdot\left(h_{n} \vec{\nabla} \lambda_{0 n}-\vec{\nabla} \phi\right)}{-\vec{\mu} \cdot h_{n} \vec{\nabla} \lambda_{0 n}}$

where $q_{n}$ is the Poissonian induced charge of the heterogeneous system, with $n=1,2$ or 3 depending on within which layer the void is located.

With the planar geometry, we may regard the dipole moment as being directed away from the coordinate origin in the positive $z$ direction. This moment can then be expressed as

$\vec{\mu}=\mu \vec{e}$

Undertaking the vector operations, (18) simplifies to give

$\frac{q_{P n}}{q_{n}}=1-\frac{\mathrm{d} \phi / \mathrm{d} z}{h_{n} \mathrm{~d} \lambda_{0 n} / \mathrm{d} z}$

Hence using (14), (15), (16) and (17) we obtain for a void in medium 1

$\frac{q_{P 1}}{q_{1}}=1-\frac{A}{\varepsilon_{2} \varepsilon_{3} h_{1} d}$

$\frac{q_{P 2}}{q_{2}}=1-\frac{A}{\varepsilon_{1} \varepsilon_{3} h_{2} d}$

for a void in layer 2, and similarly for a void in layer 3

$\frac{q_{P 3}}{q_{3}}=1-\frac{A}{\varepsilon_{1} \varepsilon_{2} h_{3} d}$

As (21), (22) and (23) contain $h$, then this implies that $q_{P n} / q_{n}$ is dependent on the void geometry. In this study we will assume the void shape as spherical, in which case we have

$h=\frac{3 \varepsilon_{r}}{1+2 \varepsilon r}$

where $\varepsilon_{r}$ is the relative permittivity of the dielectric layer containing the void.

The variation of $q_{P n} / q_{n}$ with $\varepsilon_{2} / \varepsilon_{1}$ is shown in Fig.1, with $\varepsilon_{r}=4$ and for 
several values of $\varepsilon_{13}$. On this occasion 3 layers of equal thickness $(d / 3)$ are considered.

Taking $\varepsilon_{2} / \varepsilon_{1}=1$ as a reference condition, we see that $q_{P n}$ either increases or decreases depending upon whether the void is located in a medium of lower or higher permittivity. This behaviour is similar to that of a two-layer system, viz. dependent on the value of $\varepsilon_{2} / \varepsilon_{1}$. However, the actual magnitude of $q_{P n}$ is now influenced by the relative permittivity of the third layer, $\varepsilon_{\mathrm{r} 3}$. This response can be readily appreciated since, for $\varepsilon_{2} / \varepsilon_{1}=1$, the 3-1ayer system becomes effectively a 2-layer system.

As $\varepsilon_{r}=4$, then for $\varepsilon_{2} / \varepsilon_{1}=1$ and $\varepsilon_{r 3}=$ 4 we have a homogeneous system. Thus with a spherical void, $q_{P n} / q_{n}=0.25$, see Fig.1. Consequently for $\varepsilon_{r_{3}}>4$, the void is in a (combined) medium of lower permittivity, leading to an increased $q_{P n} / q_{n}$, 'i.e. $>0.25$, see

Fig.1. Likewise a decrease occurs when the void is located in a medium of greater permittivity, i.e. when $\varepsilon_{r_{3}}<$ 4, $q_{P n} / q_{n}<0.25$. The latter reduction is more pronounced than the corresponding increase.

For a fixed void location $q_{\mu}$ is dependent only on $\vec{\mu}$, which is assumed constant in this study. Hence variations in $q_{P}$ will be directly reflected in the magnitude of the Poissonian induced charge $q$, see (1).

\section{CONCLUSIONS}

In comparison to a 2-layer dielectric system, the introduction of a third dielectric layer can significantly modify the induced charge component associated with changes in bulk dielectric polarization. The nature of these changes is dependent on the relative permittivities of the 3 dielectrics.-Consequently, for equipment employing multiple dielectrics, a correct interpretation of $P D$ transients, with respect to discharge phenomena within a void, does not appear to be possible without full knowledge of the permittivities involved.

\section{REFERENCES}

[1] A. Pedersen, G. C. Crichton and I.W. McAllister, "The Functional Relation between Partial Discharges and Induced Charge", IEEE Trans. Diel. \& Elect. Insul., 2, pp.535-543, 1995.

[2] I. W. McAllister \& G. C. Crichton "Influence of Bulk Dielectric Polarization upon Partial Discharge Transients: Effect of Heterogeneous Dielectric Geometry", IEEE Trans. Diel. \& Elect. Insul. accepted for publication 1998.

[3] A. Pedersen, G. C. Crichton and I. W. McAllister, "Partial Discharge Detection : Theoretical and Practical Aspects", IEE Proc.Sci. Meas. Techno1., 142, pp.2936,1995 .

[4] A. Pedersen, G. C. Crichton and I.W. McAllister, "The Theory and Measurement of Partial Discharge Transients", IEEE Trans. Elect. Insul., 26, pp.487-497, 1991 .

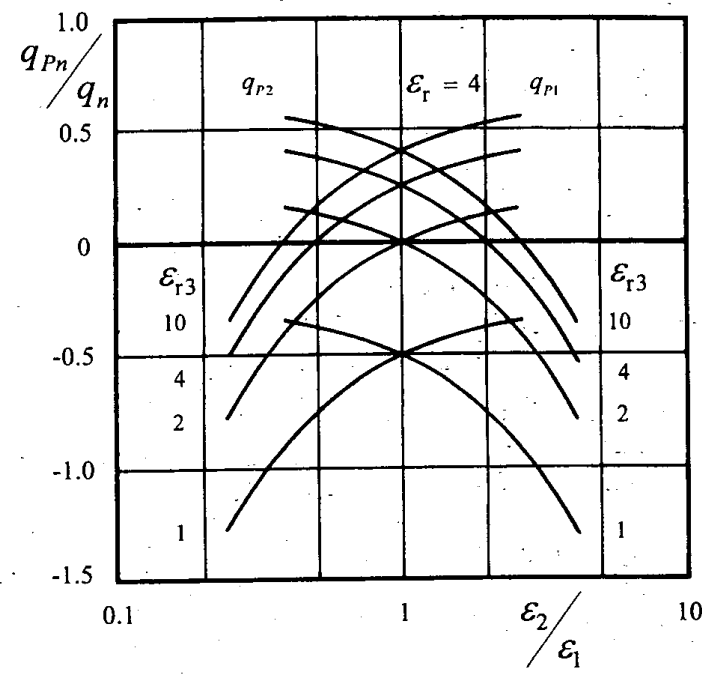

Fig.1 Variation of the polarization component of the Poissonian induced charge. $(s=t-s=d-t=d / 3)$. . 